\title{
Degradation of antifungal anthraquinone compounds is a probable physiological role of DyP secreted by Bjerkandera adusta
}

\author{
Kanako Sugawara , Etsuno Igeta ${ }^{2}$ Yoshimi Amano ${ }^{1}$, Mayuko Hyuga ${ }^{1}$ and Yasushi Sugano ${ }^{1,2^{*}}$ (D)
}

\begin{abstract}
Alizarin is an anti-fungal compound produced by the plant, Rubia tinctorum. The parasitic fungus Bjerkandera adusta Dec 1 was cultured in potato dextrose (PD) medium with or without alizarin. Alizarin was a good substrate for the dye-decolorizing peroxidase (DyP) from B. adusta Dec 1 and hampered B. adusta growth at the early stage of plate culture. During liquid shaking culture, DyP activity in cultures supplemented with $100 \mu \mathrm{M}$ alizarin was greater than that in controls cultured without alizarin. In particular, DyP activity per dry cell mass increased approximately 3.5-, 3.1-, and 2.9-fold at 24, 30, and $36 \mathrm{~h}$ after inoculation, respectively, compared with control cultures. These data suggest that alizarin stimulates the expression of DyP. Interestingly, alizarin rapidly decomposed at an early stage in culture (24-42 h) in PD medium supplemented with $100 \mu \mathrm{M}$ alizarin. Thus, alizarin appears to induce DyP expression in $B$. adusta Dec 1 , and this DyP, in turn, rapidly degrades alizarin. Collectively, our findings suggest that the physiological role of DyP is to degrade antifungal compounds produced by plants.
\end{abstract}

Keywords: DyP, Dye-decolorizing peroxidase, Basidiomycetes, Bjerkandera adusta, Antifungal anthraquinone compounds, Physiological role

\section{Introduction}

Bjerkandera adusta, a basidiomycete belonging to the family Polyporaceae, is a white rot fungus that parasitizes certain trees, resulting in lignin degradation. Notably, B. adusta Dec 1 (FERM P-15348) decolorizes kraft pulp lignin (Shintani et al. 2002). Manganese peroxidase $(\mathrm{MnP})$ and dye decolorizing peroxidase $(\mathrm{DyP})$ have been detected during culture of B. adusta Dec 1, but lignin peroxidase (LiP) and laccase have not. Another noteworthy characteristic is that Dec 1 degrades xenobiotics such as synthetic anthraquinone dyes and secretes a versatile peroxidase (VP) in addition to DyP during culture (Kim et al. 1995; Sugano et al. 2000, 2006, 2009; Gomi et al. 2011). However, VP activities toward several anthraquinone compounds are only $2-20 \%$ of those

\footnotetext{
*Correspondence: suganoy@fc.jwu.ac.jp

${ }^{1}$ Department of Chemical and Biological Sciences, Faculty of Science, Japan Women's University, 2-8-1 Mejirodai, Bunkyo-ku, Tokyo 112-8681, Japan

Full list of author information is available at the end of the article
}

of DyP, clearly indicating that $\mathrm{DyP}$ of $B$. adusta is the main degrader of anthraquinone compounds (Sugano et al. 2006). On the other hand, because synthetic dyes are never true substrates, the physiological role of DyP is unknown and thus remains an essential question.

DyP of B. adusta is a member of a large family of DyPtype peroxidases that is subdivided in three classes, P, I and $\mathrm{V}$, according to their tertiary structural homology (Yoshida and Sugano 2015). A unique feature of this family is that their characteristics, including cellular localization and primary structures, vary widely and differ considerably among the three classes (Sugano et al. 2007; Sugano 2009; Yoshida and Sugano 2015). Members of class $\mathrm{P}$, which have the smallest molecular size among the three classes, are characterized by general peroxidase activity, but low anthraquinone degradation activity (Ahmad et al. 2011; Roberts et al. 2011; Singh et al. 2012; Rahmanpour and Bugg 2013; Yoshida and Sugano 2015). Members of class I are intermediate in size, and some show moderate decolorizing activity toward 
anthraquinone dyes (Ahmad et al. 2011; Roberts et al. 2011; Santos et al. 2014). Of the three classes, members of class $\mathrm{V}$, which are distributed among both prokaryotes and eukaryotes, have the largest molecular size (Yoshida and Sugano 2015; Sugawara et al. 2017). Notably, most members of class $\mathrm{V}$ are produced and secreted by basidiomycetes (Johjima et al. 2003; Scheibner et al. 2008; Liers et al. 2010, 2013; Kellner et al. 2014), predominantly white rot fungi such as $B$. adusta, and show high decolorizing activity toward anthraquinone dyes (Sugano et al. 2000; Liers et al. 2010, 2013; Salvachúa et al. 2013). From another standpoint, the class V DyP-type peroxidases, AjP I, AjP II, EglDyP and MepDyP, from basidiomycetes are secreted outside the cell and degrade non-phenolic lignin model compounds through their peroxidase activity (Liers et al. 2010, 2013). This suggests that these proteins function in the oxidative degradation of lignin. Consistent with this, widespread transcript-level expression of DyP-type peroxidases has been confirmed in almost all samples of fungi from forest floor habitats (Kellner et al. 2014). However, the lignin-degrading activity of DyP-type peroxidases from basidiomycetes is at most $4 \%$ of that of LiP from Phanerochaete chrysosporium (Liers et al. 2013; Linde et al. 2015). DypB from Rhodococcus jostii has been reported to degrade lignin in the presence of $\mathrm{Mn}(\mathrm{II})$, but its activity is low compared with that of fungal lignin-degrading enzymes (Ahmad et al. 2011; Brown et al. 2012; Linde et al. 2015).

In contrast, LiP, MnP, and VP are well known as major contributors to lignin degradation in white rot fungi (Wariishi et al. 1989; Camarero et al. 1999; Johjima et al. 1999; Pollegioni et al. 2015). Actually, some basidiomycetes that express LiP and MnP, but lack DyP-type peroxidases, such as $P$. chrysosporium, exhibit high lignin-degradation activity (Korripally et al. 2015). These observations suggest the possibility that the true physiological role of DyP from basidiomycetes is something other than lignin degradation, prompting us to focus on anthraquinone compounds as potential substrates. As mentioned above, DyP readily degrades anthraquinone compounds (Sugano et al. 2000; Ogola et al. 2009; Liers et al. 2010, 2013; Salvachúa et al. 2013). Interestingly, plants, including trees, express a number of anthraquinone compounds that serve antifungal functions. One such representative antifungal compound is alizarin, produced by the evergreen perennial, Rubia tinctorum (Manojlovic et al. 2005; Jara et al. 2017). Generally, white rot fungi belonging to the class basidiomycetes are best known for their selective parasitism of old or dead trees. They rarely grow on young or healthy trees because these trees generate phytoalexin, which serves to protect against infection (Wijnsma et al. 1985). In contrast, $B$. adusta is often observed to parasitize some living trees in a forest (Berry and Lombard 1978). This raises the question of how B. adusta evades the defense of plants.

In this study, we focused on the ability of DyP to degrade the anti-fungal anthraquinone compound, alizarin (Manojlovic et al. 2005), and further considered the possibility that DyP degrades natural anthraquinone compounds, such as some phytoalexins (Wijnsma et al. 1985). If this were true, it would help explain how some basidiomycetes parasitize living trees, despite the fact that these trees produce antifungal compounds, such as anthraquinones (Amaral et al. 2013). Here, we found that alizarin stimulated the secretion of DyP by the white rot fungus $B$. adusta. This is the first report that DyP truly degrades an antifungal anthraquinone compound in plants, a finding that could set the stage for resolving questions regarding interactions between fungi and plants.

\section{Materials and methods Microorganisms, media, DyP, and chemical reagents} The spores of $B$. adusta Dec 1, previously isolated by us, were kept in $25 \%$ glycerol at $-80{ }^{\circ} \mathrm{C}$ (Kim et al. 1995). Potato dextrose (PD) medium was prepared as described previously (Sugano et al. 2006). Purified recombinant DyP in Aspergillus oryzae was prepared using a previously reported method (Sugano et al. 2000). Alizarin and Remazol brilliant blue R (RB19) were purchased from Wako Chemical Co. (Tokyo, Japan). All other reagents were of analytical grade unless otherwise specified.

\section{Culture}

A suspension of $B$. adusta Dec 1 spores was inoculated onto PD agar plates $(10 \mu \mathrm{l} /$ plate $)$ and incubated for 12 days at $29^{\circ} \mathrm{C}$. Mycelia from six plates were collected and suspended in $10 \mathrm{ml}$ of sterilized, distilled water. The suspension was agitated vigorously for $7 \mathrm{~min}$ and filtered through gauze, after which 8-ml aliquots of filtrate, which included $1.0 \times 10^{7}-10^{8}$ spores $/ \mathrm{ml}$, were inoculated into $250 \mathrm{ml}$ of PD containing $100 \mu \mathrm{M}$ alizarin in shaking flasks. Alizarin was excluded from medium in control cultures. Cultures were grown at $29{ }^{\circ} \mathrm{C}$ with shaking at $140 \mathrm{rpm}$.

\section{Growth rate of $B$. adusta Dec 1 under plate culture conditions}

PD agar plates $(8.5 \mathrm{~cm} \phi)$ containing five different alizarin concentrations $(0,1,3.2,10$ and $32 \mu \mathrm{M})$ were prepared. A suspension of $B$. adusta Dec 1 spores was inoculated onto the center of each plate $(10 \mu \mathrm{l} /$ plate $)$ and incubated at $29{ }^{\circ} \mathrm{C}$. The diameters of mycelia on plates were measured periodically. 


\section{Sampling and preparation of dry cell mass}

Thirty milliliters of culture broth were periodically harvested from shaking flask cultures. The sampled broth was centrifuged at $1500 \times g\left(4{ }^{\circ} \mathrm{C}, 1 \mathrm{~h}\right)$ in conical tubes, and the supernatant and precipitate were separated. The collected supernatant was used to assay alizarin concentration and enzymatic activity.

A membrane filter method was used to measure dry cell mass. Briefly, after harvesting and centrifuging $30 \mathrm{ml}$ of culture broth and separating the supernatant and precipitate as described above, the precipitates were re-suspended in $30 \mathrm{ml}$ of $1 \mathrm{mM} \mathrm{CaCl}_{2}$ and then treated with $10 \mu \mathrm{l}$ of $\alpha$-amylase (Novozymes) at $70{ }^{\circ} \mathrm{C}$ for $1 \mathrm{~h}$. Thereafter, the suspension was collected on a cellulose acetate membrane filter (pore size, $0.8 \mu \mathrm{m}$ ), which was subsequently dried at $80{ }^{\circ} \mathrm{C}$ for $24 \mathrm{~h}$. The dry cell mass was then calculated from the total weight minus the weight of the membrane filter.

\section{HPLC analysis of alizarin}

Saccharides in culture broth were removed by adding $1 \mathrm{ml}$ of ethanol to $500 \mu \mathrm{l}$ of the culture supernatant. The mixture was centrifuged at $4{ }^{\circ} \mathrm{C}(11,000 \times g$, $10 \mathrm{~min})$, after which the supernatant was filtered through a cellulose acetate membrane (pore size, $0.45 \mu \mathrm{m})$. The amount of alizarin in the resulting filtrate was measured by high-performance liquid chromatography (HPLC) using a Capcell Pak C8 column $(4.6 \mathrm{~mm}$ $\phi \times 250 \mathrm{~mm}$ ) with a flow rate of $1.0 \mathrm{ml} / \mathrm{min}$. Alizarin was eluted with a $45: 55(\mathrm{v} / \mathrm{v})$ solution of $95 \%$ acetonitrile: $20 \mathrm{mM}$ ammonium formate $(\mathrm{pH} 3.0)$, and was detected at a wavelength of $254 \mathrm{~nm}$.

\section{Assay of DyP activity}

Thirty milliliters of supernatant from the culture broth was concentrated to $50 \mu \mathrm{l}$ by ultrafiltration (exclusion size, $30 \mathrm{kDa}$ ), to which was added $450 \mu \mathrm{l}$ of $25 \mathrm{mM}$ citrate buffer ( $\mathrm{pH}$ 5.5). The resulting solution was then reconcentrated to $50 \mu \mathrm{l}$. This operation was repeated twice, and the final volume was adjusted to $300 \mu \mathrm{l}$ with the same buffer. This solution was prepared for assay of DyP activity at $30{ }^{\circ} \mathrm{C}$ by adding $15 \mu \mathrm{l}$ of $20 \mathrm{mM}$ RB19 (DyP substrate), $750 \mu \mathrm{l}$ of $100 \mathrm{mM}$ citrate buffer (pH 3.2), and $1920 \mu \mathrm{l}$ of distilled water in a 3-ml cuvette. The reaction was initiated by adding $15 \mu \mathrm{l}$ of $40 \mathrm{mM} \mathrm{H}_{2} \mathrm{O}_{2}$, and decolorization of RB19 was monitored spectrophotometrically at $593 \mathrm{~nm}$. One unit of DyP activity was defined as the amount of enzyme required to decolorize $1 \mu \mathrm{mol}$ of RB19 $\left(\varepsilon_{593}=9100 \mathrm{M}^{-1} \mathrm{~cm}^{-1}\right)$ in the reaction mixture in $1 \mathrm{~min}$ at $30^{\circ} \mathrm{C}$.

\section{Degradation of alizarin by recombinant DyP}

Alizarin is poorly soluble in water and thus was prepared as a $20 \mathrm{mM}$ solution in dimethyl sulfoxide (DMSO). Reaction mixtures were prepared in a 3-ml cuvette by combining purified, recombinant DyP [(Sugano et al. 2000); final concentration, $1.55 \mathrm{nM}$ ], $18 \mu \mathrm{l}$ of $20 \mathrm{mM}$ alizarin (final concentration, $125 \mu \mathrm{M}$ ), $750 \mu \mathrm{l}$ of $100 \mathrm{mM}$ citrate buffer (pH 3.2) and $582 \mu \mathrm{l}$ of DMSO (final concentration, 20\%), and adjusting the total volume to $2985 \mu \mathrm{l}$ with distilled water. The reaction was initiated by adding $15 \mu \mathrm{l}$ of $40 \mathrm{mM} \mathrm{H}_{2} \mathrm{O}_{2}$, and decolorization was monitored spectrophotometrically at $427 \mathrm{~nm}$. The alizarin-degrading activity of DyP was compared with that of RB19 under the same reaction conditions (i.e., containing 20\% DMSO).

The optimum $\mathrm{pH}$ for recombinant DyP activity toward alizarin was determined using a citrate buffer $(25 \mathrm{mM})$ covering a $\mathrm{pH}$ range of 3.0 to 5.5. Reaction conditions, except for buffer $\mathrm{pH}$, were the same as above.

\section{Effects of $\mathrm{pH}$ on alizarin degradation in liquid cultures containing DyP}

Twenty milliliters of culture broth and cells were periodically harvested from shaking flask cultures. The $\mathrm{pH}$ of culture broth was immediately measured and adjusted to 3.2. Thereafter, cultures were incubated at $30{ }^{\circ} \mathrm{C}$ for $30 \mathrm{~min}$ and centrifuged to remove cells. The alizarin concentration in the resulting supernatants was analyzed as described above. Controls were not $\mathrm{pH}$-adjusted.

\section{Results}

\section{Degradation activity of DyP toward alizarin}

At DMSO concentrations lower than $10 \%$, alizarin did not completely dissolve in the reaction mixture. As shown in Fig. 1a, increasing the concentration of DMSO in the reaction mixture caused a gradual decrease in recombinant DyP activity. Notably, however, the degrading activity of DyP towards the general substrate RB19 and alizarin followed a similar pattern. For instance, in reaction solutions containing 20\% DMSO, the degradation activity of DyP for RB19 and alizarin was 5.5 and $4.6 \mu \mathrm{M} / \mathrm{min}$, respectively, indicating that both alizarin and RB19 are good substrates for DyP. An examination of the kinetic parameters of recombinant DyP toward alizarin were not able to yield $k_{\text {cat }}$ values because of DMSO inhibition. As shown in Fig. 1a, DMSO in the reaction mixture reduced activity by up to $23 \%$ compared to the reaction without DMSO.

We also examined the optimum $\mathrm{pH}$ for degradation of alizarin by recombinant DyP. Recombinant DyP 


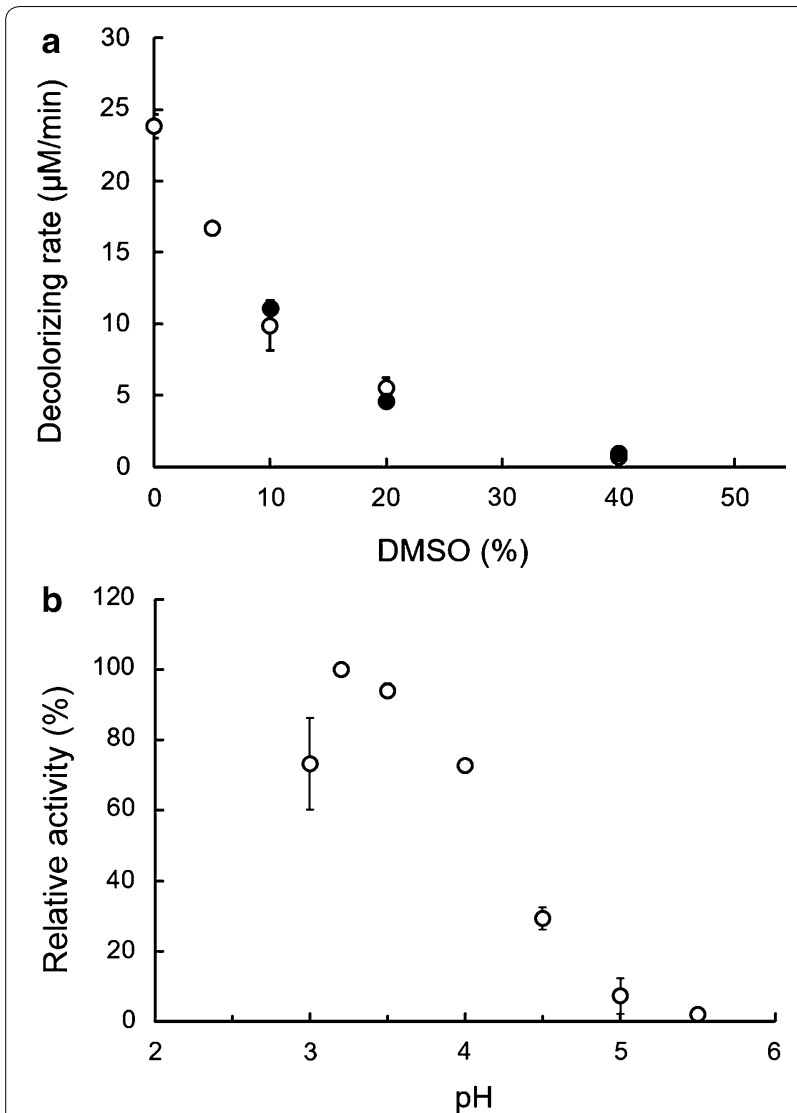

Fig. 1 Effects of solvent and pH on DyP activity. a Relationship between DyP activity and DMSO concentration. Open and closed symbols denote the degradation activity of recombinant DyP towards RB19 and alizarin, respectively. $\mathbf{b}$ pH profile of the activity of recombinant DyP toward alizarin

exhibited activity towards alizarin within the $\mathrm{pH}$ range of 3.0 to 5.0, and degraded most alizarin at $\mathrm{pH} 3.2$ (Fig. 1b). These results are consistent with $\mathrm{pH}$ profiles for RB19 degradation activity, for which the reported optimum $\mathrm{pH}$ is 3.2 (Sugano et al. 2000).

\section{B. adusta Dec 1 growth in plate cultures}

In initial experiments, we tested the effect of alizarin on $B$. adusta Dec 1 growth in plate cultures. As shown in Fig. 2, DMSO had no effect on B. adusta growth. After an initial lag phase $(\sim 25 \mathrm{~h}), B$. adusta growth, measured as an increase in mycelia diameter, increased over time at all concentrations of alizarin tested. At a relatively early point after inoculation $(69 \mathrm{~h})$, the diameters of mycelia varied depending on the concentration of alizarin, with increasing concentrations of alizarin resulting in smaller diameters (Fig. 2). However, after $101 \mathrm{~h}$, the growth rate (slope) was largely unaffected by alizarin concentration, suggesting that alizarin hampers growth at an early stage of plate culture, but not after middle stages of

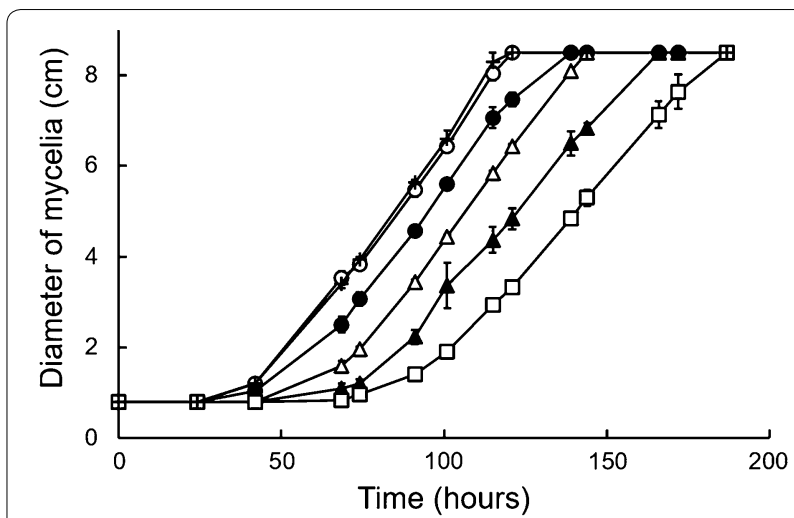

Fig. 2 Time courses of B. adusta Dec 1 mycelia growth on PD plates containing different concentrations of alizarin. Crosses, $0 \mu \mathrm{M}$ alizarin without DMSO; open circles, $0 \mu \mathrm{M}$ alizarin with 0.64\% DMSO; closed circles, $1 \mu \mathrm{M}$ alizarin; open triangles, $3.2 \mu \mathrm{M}$ alizarin; closed triangles, $10 \mu \mathrm{M}$ alizarin; open squares, $32 \mu \mathrm{M}$ alizarin

culture. Also, alizarin did not affect hyphal morphology in B. adusta Dec 1. Furthermore, the mycelia of B. adusta showed no change in color following culture with alizarin (orange-colored solution in DMSO), indicating that alizarin was not absorbed by mycelia.

\section{Time-dependent decrease in alizarin concentration in liquid shaking culture}

The time course of changes in alizarin concentration following inoculation with $100 \mu \mathrm{M}$ is shown in Fig. 3 . Interestingly, $36 \mathrm{~h}$ after inoculation, the alizarin concentration had decreased to $58 \mu \mathrm{M}$ and stabilized at a very low level $(<5 \mu \mathrm{M})$ by $60 \mathrm{~h}$. This indicates that alizarin was nearly completely degraded at an early stage in culture; if true, DyP must act efficiently at an early stage. In subsequent experiments, we sought to confirm this early-stage action, investigating effects within $48 \mathrm{~h}$ after inoculation with $100 \mu \mathrm{M}$ alizarin.

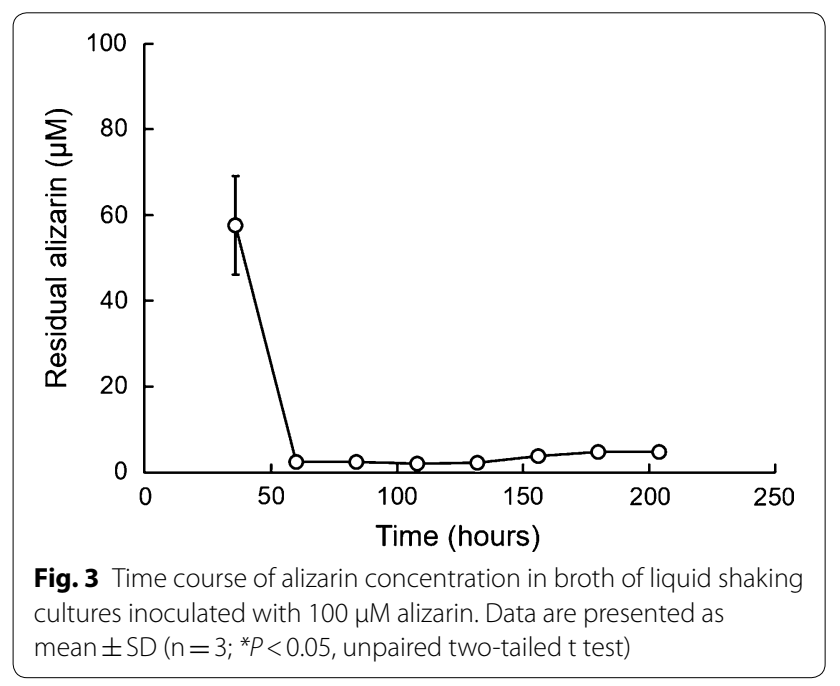




\section{B. adusta growth in liquid culture}

To further assess the effect of alizarin on B. adusta growth, we measured cell mass accumulation over time in liquid cultures. A time course of cell mass accumulation following treatment with $100 \mu \mathrm{M}$ alizarin is shown in Fig. 4a. Alizarin $(100 \mu \mathrm{M})$ caused a decrease in dry
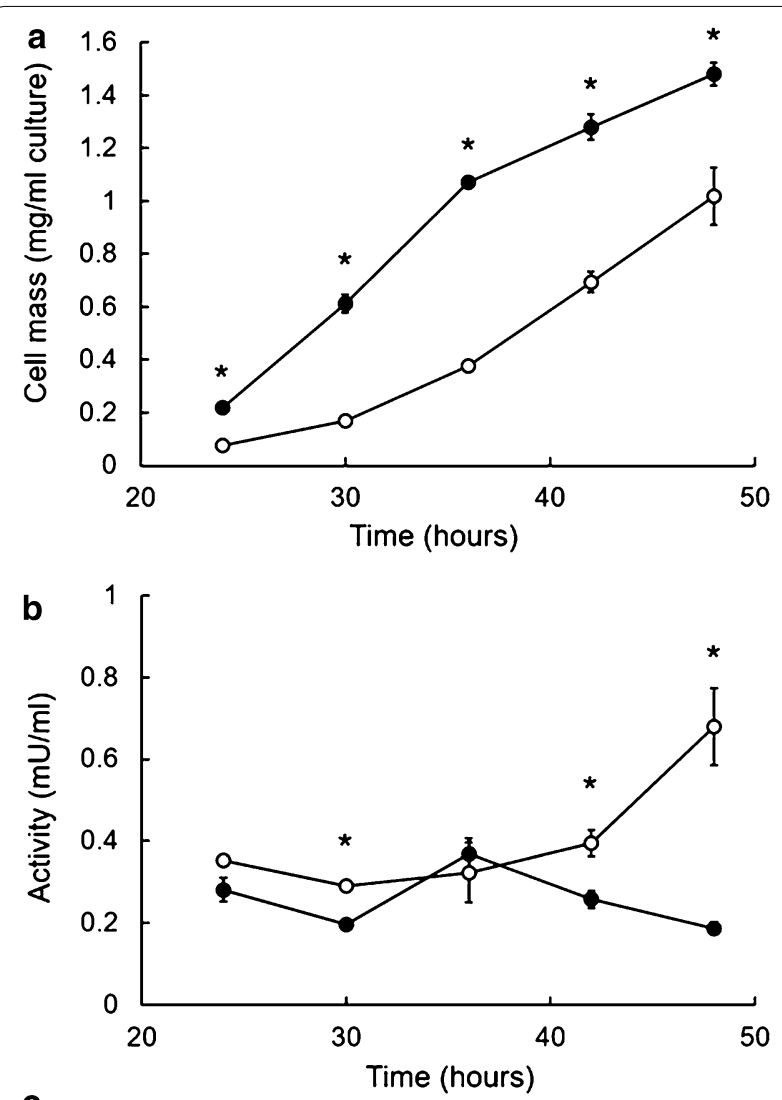

C

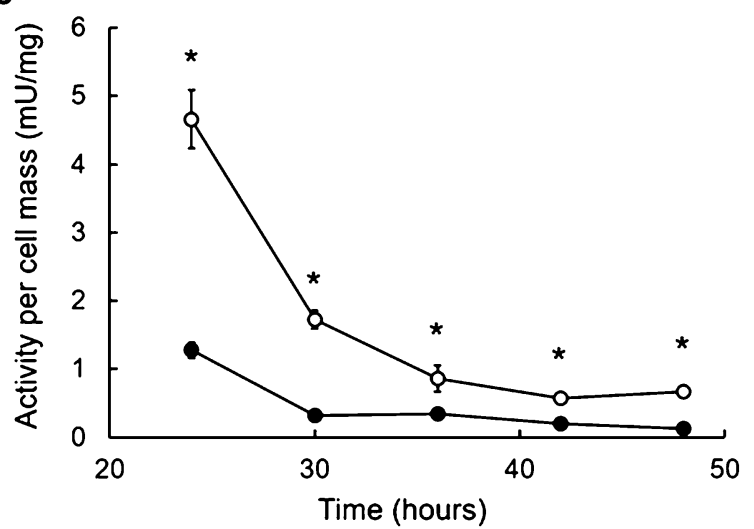

Fig. 4 Time courses of dry cell mass accumulation and DyP activity at the early stage of culture with $100 \mu \mathrm{M}$ alizarin. Open and closed symbols denote with and without alizarin, respectively. a Dry cell mass; $\mathbf{b}$ DyP activity per milliliter culture broth; $\mathbf{c}$ DyP activity per milligram dry cell mass. Data are presented as mean $\pm S D(n=3$; ${ }^{*} P<0.05$, unpaired two-tailed $t$ test) cell mass compared with control cultures without alizarin at all sampling times.

\section{DyP activity in liquid culture}

We next assessed DyP activity over time in liquid cultures of B. adusta, with or without alizarin. Time courses of DyP activity, expressed as milliunits per milliliter $(\mathrm{mU} /$ $\mathrm{ml})$ of culture broth and milliunits per milligram $(\mathrm{mU} /$ mg) dry cell mass, are shown in Fig. 4b, c, respectively. Notably, DyP activity per cell mass in the presence of alizarin was approximately 3.5-, 3.1-, and 2.9-fold higher at 24,30 , and $36 \mathrm{~h}$, respectively, than that observed in control cultures without alizarin (Fig. 4c). This suggests that DyP activity per cell in liquid cultures containing alizarin is greater than that in cultures without alizarin.

\section{Time- and pH-dependent decrease in alizarin in liquid culture}

A time course of alizarin concentration in liquid culture indicated little decrease before $24 \mathrm{~h}$ and a rapid decrease from 24 to $42 \mathrm{~h}$ after inoculation (Fig. 5, closed circles). In addition, the decrease in the $\mathrm{pH}$ of the culture broth followed the same time course as the decrease in alizarin concentration (Fig. 5, closed triangles). Given that the optimum $\mathrm{pH}$ of DyP from B. adusta Dec 1 is $\sim 3.2$, this suggests that alizarin degradation began as the $\mathrm{pH}$ in liquid culture crossed a critical threshold value, and increased rapidly as $\mathrm{pH}$ fell to within the optimal range (provided DyP is sufficiently expressed). Consistent with this, the alizarin concentration in culture broth adjusted to $\mathrm{pH} 3.2$ decreased at a constant, rapid rate from 0 to $30 \mathrm{~h}$ (Fig. 5, opened circles).

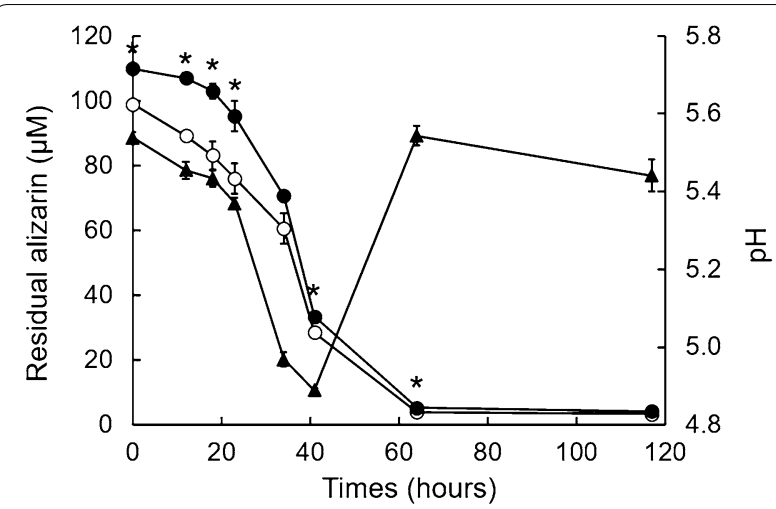

Fig. 5 Effect of pH on alizarin degradation by DyP. Time courses of alizarin concentration and $\mathrm{pH}$ values in liquid cultures inoculated with $100 \mu \mathrm{M}$ alizarin. Open and closed circles are residual alizarin concentrations at pH 3.2 (optimum pH for DyP activity) and at unadjusted $\mathrm{pH}$, respectively. Closed triangles denote the time course of $\mathrm{pH}$ values. Data are presented as mean $\pm \mathrm{SD}\left(\mathrm{n}=3 ;{ }^{*} P<0.05\right.$, unpaired two-tailed t test) 


\section{Discussion}

DyP degradation activity toward both alizarin and RB19 was similar as shown in Fig. 1, indicating that alizarin is a good substrate for DyP. Therefore, phytoalexins such as alizarin are probable candidate essential substrates. If this were true, $B$. adusta Dec 1 would grow without inhibition in medium containing alizarin. However, our experimental results suggest that the relationship between alizarin degradation and DyP activity during the culture of $B$. adusta is more complicated than this simple picture would suggest.

In plate cultures, increasing the concentration of alizarin caused growth inhibition, but this effect was limited to an early stage of culture (Fig. 2). Interestingly, after this early stage, cells continued to grow without inhibition, suggesting that alizarin is degraded during the early growth of $B$. adusta. Similarly, $100 \mu \mathrm{M}$ alizarin was almost completely degraded before $60 \mathrm{~h}$ in liquid shaking culture (Fig. 3). These results are supported by analyses of alizarin in liquid culture (Fig. 5), which showed that alizarin concentration decreased beginning at $\sim 24 \mathrm{~h}$. Alizarin showed little degradation prior to $24 \mathrm{~h}$, indicating that DyP was not effective during this period. However, DyP activity per dry cell mass in liquid culture containing $100 \mu \mathrm{M}$ alizarin was always higher than that of controls during the culture period (Fig. 4c). Notably, DyP activity per dry cell mass in alizarin-treated cultures was greater than that in controls, even at $24 \mathrm{~h}$, suggesting that alizarin had stimulated DyP expression before $24 \mathrm{~h}$ (Fig. 4c). These results suggest that alizarin induces greater DyP activity before $24 \mathrm{~h}$ in liquid culture, but DyP activity remains below the limit of detection because of the low number of cells prior to $24 \mathrm{~h}$.

Although DyP is apparently induced before $24 \mathrm{~h}$ in cultures containing alizarin, alizarin degradation had not yet started. This outcome is attributable to the $\mathrm{pH}$ value of the culture, which was unsuitable for DyP activity (Fig. 5). Until $18 \mathrm{~h}$ after inoculation, the $\mathrm{pH}$ of cultures was maintained above 5.4. Under these conditions, DyP activity toward RB19 is less than $5 \%$ of its maximum value (Sugano et al. 2000). On the other hand, from 24 to $40 \mathrm{~h}$ in culture, the $\mathrm{pH}$ decreased to $4.8-5.3$, and $\mathrm{DyP}$ exhibited $10-20 \%$ of its maximum activity towards RB19 (Sugano et al. 2000). Therefore, if the $\mathrm{pH}$ of the culture is below 5.3, this concentration of DyP $(0.4 \mathrm{mU} / \mathrm{ml})$ is probably sufficient to degrade alizarin, because DyP showed almost the same degree of degradation activity towards alizarin as it did toward the substrate RB19 (Fig. 1).

The difference in sensitivity of DyP for alizarin between plate cultures and liquid cultures is likely attributable to differences in the diffusion of alizarin. In agarose gels, alizarin diffuses slowly compared with that in solution. Therefore, during degradation by $B$. adusta, the concentration of alizarin in the plate becomes less homogeneous than is the case in liquid culture. As a consequence, the degradation rate of alizarin is faster in liquid cultures than in plate cultures for a given starting concentration. In nature, the environment on plates might be more similar to that on trees. Therefore, B. adusta would be predicted parasitize trees slowly over time.

Collectively, these results suggest that, upon recognition of an antifungal anthraquinone compound in the environment, $B$. adusta Dec 1 secretes DyP, which then degrades the compounds first before initiating growth. After DyP has sufficiently decomposed the anthraquinone compound to prevent its growth-inhibitory effects, B. adusta begins to grow. This relationship accounts for the time lag for the initiation of growth. Thus, we propose that a probable physiological role of the $B$. adusta DyP-type peroxidase is to destroy the antifungal system of plants-a survival mechanism that $B$. adusta exploits in nature to parasitize trees. Notably, this idea is distinct from the concept of DyP involvement in lignin degradation. A $d y p$ knockdown or knockout strain would provide a tool for directly testing this idea. Unfortunately, it has proved difficult to construct these strains because B. adusta has several isozymes of DyP. Moreover, genome-editing techniques for gene knockout in fungi are available only for a subset of yeast and ascomycetes (DiCarlo et al. 2013; Matsu-ura et al. 2015). However, genome editing in basidiomycete was recently reported (Schuster et al. 2016), an encouraging development for the application of these powerful genetic approaches. Although genome editing in $B$. adusta remains challenging because so few applications have been reported, we will attempt to establish a knockout mutant strain in the future. Elucidating the detailed mechanism by which $B$. adusta degrades alizarin might pave the way to answering questions regarding interactions between fungi and plants.

\section{Abbreviations \\ DyP: dye-decolorizing peroxidase; rDyP: recombinant DyP; LiP: lignin peroxi- dase; MnP: manganese peroxidase; VP: versatile peroxidase; RB19: Remazol brilliant blue $\mathrm{R}$.}

\section{Authors' contributions}

KS performed the experiments, all data analysis and wrote the original draft. El and $\mathrm{MH}$ performed the experiments and partial data analysis. YA designed the partial experiments. YS supervised the project and revised the manuscript. All authors read and approved the final manuscript.

\section{Author details}

${ }^{1}$ Department of Chemical and Biological Sciences, Faculty of Science, Japan Women's University, 2-8-1 Mejirodai, Bunkyo-ku, Tokyo 112-8681, Japan. ${ }^{2}$ Division of Material and Biological Sciences, Graduate School of Science, Japan Women's University, 2-8-1 Mejirodai, Bunkyo-ku, Tokyo 112-8681, Japan.

Competing interests

The authors declare that they have no competing interests. 


\section{Availability of data and materials}

All data analyzed throughout this study are shown in this article. The strain Dec 1 was deposited to Biological Resource Center, NITE (NBRC) as the registry number as FERM P-15348.

\section{Consent for publication}

Not applicable.

\section{Ethics approval and consent to participate}

Not applicable.

\section{Funding}

This research was supported in part by Grants in Network Joint Research Center for Materials and Devices Nos. 20161166, 20183045 and JSPS KAKENHI Grant No. 26450132.

\section{Publisher's Note}

Springer Nature remains neutral with regard to jurisdictional claims in published maps and institutional affiliations.

Received: 20 November 2018 Accepted: 16 April 2019

Published online: 23 April 2019

\section{References}

Ahmad M, Roberts JN, Hardiman EM, Singh R, Eltis LD, Bugg TDH (2011) Identification of DypB from Rhodococcus jostii RHA1 as a lignin peroxidase. Biochemistry 50:5096-5107

Amaral LF, Moriel P, Foglio MA, Mazzola PG (2013) Caryocar brasiliense supercritical $\mathrm{CO}_{2}$ extract possesses antimicrobial and antioxidant properties useful for personal care products. BMC Complement Altern Med 14:73. https://doi.org/10.1186/1472-6882-14-73

Berry FH and Lombard FF (1978) Basidiomycetes associated with decay of living oak trees. Forest Service Research Paper NE-413

Brown ME, Barros T, Chang MCY (2012) Identification and characterization of a multifunctional dye peroxidase from a lignin-reactive bacterium. ACS Chem Biol 7:2074-2081

Camarero S, Sarkar S, Ruiz-Dueñas FJ, Martínez MJ, Martínez AT (1999) Description of a versatile peroxidase involved in the natural degradation of lignin that has both manganese peroxidase and lignin peroxidase substrate interaction sites. J Biol Chem 274:10324-10330

DiCarlo JE, Norville JE, Mali P, Rios X, Aach J, Church GM (2013) Genome engineering in Saccharomyces cerevisiae using CRISPR-Cas systems. Nucleic Acids Res 41:4336-4343

Gomi N, Yoshida S, Matsumoto K, Okudomi M, Konno H, Hisabori T, Sugano Y (2011) Degradation of the synthetic dye amaranth by the fungus Bjerkandera adusta Dec 1: inference of the degradation pathway from an analysis of decolorized products. Biodegradation 22:1239-1245

Jara C, Leyton M, Osorio M, Silva V, Fleming F, Paz M, Madrid A, Mellao M (2017) Antioxidant, phenolic and antifungal profiles of Acanthus mollis. Nat Prod Res 31:2325-2328. https://doi.org/10.1080/14786419.2017.1299726

Johjima T, Itoh N, Kabuto M, Tokimura F, Nakagawa T, Wariishi H, Tanaka H (1999) Direct interaction of lignin and lignin peroxidase from Phanerochaete chrysosporium. Proc Natl Acad Sci USA 96:1989-1994

Johjima T, Ohkuma M, Kudo T (2003) Isolation and cDNA cloning of novel hydrogen peroxide-dependent phenol oxidase from the basidiomycete Termitomyces albuminosus. Appl Microbiol Biotechnol 61:220-225

Kellner H, Luis P, Pecyna MJ, Barbi F, Kapturska D, Kruger D, Zak DR, Marmeisse R, Vandenbol M, Hofrichter M (2014) Widespread occurrence of expressed fungal secretory peroxidases in forest soils. PLoS ONE 9:e95557

Kim SJ, Ishikawa K, Hirai M, Shoda M (1995) Characteristics of a newly isolated fungus, Geotrichum candidum Dec 1, which decolorizes various dyes. J Ferment Bioeng 79:601-607

Korripally P, Hunt CG, Houtman CJ, Jones DC, Kitin PJ, Cullen D, Hammel KE (2015) Regulation of gene expression during the onset of ligninolytic oxidation by Phanerochaete chrysosporium on spruce wood. Appl Environ Microbiol 81:7802-7812

Liers C, Bobeth C, Pecyna M, Ullrich R, Hofrichter M (2010) DyP-like peroxidases of the jelly fungus Auricularia auricula-judae oxidize nonphenolic lignin model compounds and high-redox potential dyes. Appl Microbiol Biotechnol 85:1869-1879

Liers C, Pecyna MJ, Kellner H, Worrich A, Zorn H, Steffen KT, Hofrichter M, Ullrich R (2013) Substrate oxidation by dye-decolorizing peroxidases (DyPs) from wood- and litter-degrading agaricomycetes compared to other fungal and plant heme-peroxidases. Appl Microbiol Biotechnol 97:5839-5849

Linde D, Ruiz-Dueñas FJ, Fernández-Fueyo E, Guallar V, Hammel KE, Pogni R, Martínez AT (2015) Basidiomycete DyPs: genomic diversity, structuralfunctional aspects, reaction mechanism and environmental significance. Arch Biochem Biophys 574:66-74

Manojlovic NT, Solujic S, Sukdolak S, Milosev M (2005) Antifungal activity of Rubia tinctorum, Rhamnus frangula and Caloplaca cerina. Fitoterapia 76:244-246

Matsu-ura T, Baek M, Kwon J, Hong C (2015) Efficient gene editing in Neurospora crassa with CRISPR technology. Fungal Biol Biotechnol 2:4

Ogola HJO, Kamiike T, Hashimoto N, Ashida H, Ishikawa T, Shibata H, Sawa Y (2009) Molecular characterization of a novel peroxidase from the cyanobacterium Anabaena sp. strain PCC 7120. Appl Environ Microbiol 75:7059-7518

Pollegioni L, Tonin F, Rosini E (2015) Lignin-degrading enzymes. FEBS J 282:1190-1213

Rahmanpour R, Bugg TDH (2013) Assembly in vitro of Rhodococcus jostii RHA1 encapsulin and peroxidase DypB to form a nanocompartment. FEBS J 280:2097-2104

Roberts JN, Singh R, Grigg JC, Murphy MEP, Bugg TDH, Eltis LD (2011) Characterization of dye-decolorizing peroxidases from Rhodococcus jostii RHA1. Biochemistry 50:5108-5119

Salvachúa D, Prieto A, Martínez AT, Martínez MJ (2013) Characterization of a novel dye-decolorizimg peroxidase (DyP)-type enzyme from Irpex lacteus and its application in enzymatic hydrolysis of wheat straw. Appl Environ Microbiol 79:4316-4324

Santos A, Mendes S, Brissos V, Martins LO (2014) New dye-decolorizing peroxidases from Bacillus subtilis and Pseudomonas putida MET94: towards biotechnological applications. Appl Microbiol Biotechnol 98:2053-2065

Scheibner M, Hulsdau B, Zelena K, Nimtz M, de Boer L, Berger RG, Zorn H (2008) Novel peroxidases of Marasmius scorodonius degrade beta-carotene. Appl Microbiol Biotechnol 77:1241-1250

Schuster M, Schweizer G, Reissmann S, Kahmann R (2016) Genome editing in Ustilago maydis using the CRISPR-Cas system. Fungal Genet Biol 89:3-9

Shintani N, Sugano Y, Shoda M (2002) Decolorization of kraft pulp lignin bleaching effluent by a newly isolated fungus, Geotrichum candidum Dec 1. J Wood Sci 48:402-408

Singh R, Grigg JC, Armstrong Z, Murphy MEP, Eltis LD (2012) Distal heme pocket residues of $B$-type dye-decolorizing peroxidase: arginine but not aspartate is essential for peroxidase activity. J Biol Chem 287:10623-10630

Sugano Y (2009) DyP-type peroxidases comprise a novel heme peroxidase family. Cell Mol Life Sci 66:1387-1403

Sugano Y, Nakano R, Sasaki K, Shoda M (2000) Efficient heterologous expression in Aspergillus oryzae of a unique dye-decolorizing peroxidase, DyP, of Geotrichum candidum Dec 1. Appl Environ Microbiol 66:1754-1758

Sugano Y, Matsushima Y, Shoda M (2006) Complete decolorization of the anthraquinone dye Reactive blue 5 by the concerted action of two peroxidases from Thanatephorus cucumeris Dec 1. Appl Microbiol Biotechnol 73:862-871

Sugano Y, Muramatsu R, Ichiyanagi A, Sato T, Shoda M (2007) DyP, a unique dye-decolorizing peroxidase, represents a novel heme peroxidase family: ASP171 replaces the distal histidine of classical peroxidases. J Biol Chem 282:36652-36658

Sugano Y, Matsushima Y, Tsuchiya K, Aoki H, Hirai M, Shoda M (2009) Degradation pathway of an anthraquinone dye catalyzed by a unique peroxidase DyP from Thanatephorus cucumeris Dec 1. Biodegradation 20:433-440 
Sugawara K, Nishihashi Y, Narioka T, Yoshida T, Morita M, Sugano Y (2017) Characterization of a novel DyP-type peroxidase from Streptomyces avermitilis. J Biosci Bioeng 123:425-430

Wariishi H, Dunford HB, MacDonald ID, Gold MH (1989) Manganese peroxidase from the lignin-degrading basidiomycete Phanerochaete chrys-

osporium. Transient state kinetics and reaction mechanism. J Biol Chem 264:3335-3340
Wijnsma R, Go JT, van Weerden IN, Harkes PA, Verpoorte R, Baerheim SA (1985) Anthraquinones as phytoalexins in cell and tissue cultures of Cinchona spec. Plant Cell Rep 4:241-244

Yoshida T, Sugano Y (2015) A structural and functional perspective of DyP-type peroxidase family. Arch Bioch Biophys 574:49-55
Submit your manuscript to a SpringerOpen ${ }^{\circ}$ journal and benefit from:

- Convenient online submission

- Rigorous peer review

- Open access: articles freely available online

- High visibility within the field

- Retaining the copyright to your article

Submit your next manuscript at springeropen.com 